\title{
Implementation of Human Robot Interaction on UDOO Board
}

\author{
Min Raj Nepali, Priyanka C. Karthik, Jharna Majumdar \\ Nitte Meenakshi Institute of Technology, Bangalore
}

\begin{tabular}{l} 
Article Info \\
\hline Article history: \\
Received Nov 15, 2015 \\
Revised Jan 28, 2016 \\
Accepted Feb 19, 2016 \\
\hline Keyword: \\
Controller \\
Cross- Platform Programming \\
Dynamixel \\
Embedded Platform Graphical User \\
Interface
\end{tabular}

\begin{abstract}
Advanced Robot for Interactive Application (ARIA) is a Humanoid Robotic Head which is capable of mimicking Various Human Facial Expressions. Much work has been done on Implementation of Humanoid Robotic Head with High end systems and Personal Computers (PCs). This paper presents the essential elements necessary for the implementation of Advanced Robot for Interactive Application (ARIA) on UDOO Board. The main aim of the Project was to develop a control system and Graphical User Interface (GUI) for ARIA to deliver real time human facial expressions using embedded board. Humanoid Robotic Head which is capable of mimicking Human Facial Expressions in Real time. Implementation of ARIA involved careful selection of Embedded Board, actuators, control algorithms, motor drivers, operating system, communication protocols, and programming languages. The Board contains a Quad Core A9 Processor and a Controller embedded on it, which are interconnected. In this project the controller is dedicated to control micro servo motors which are controlling eyes, eyebrows and eyelids movements whereas the Processor Handles the Dynamixel motors, GUI and different communication modules.
\end{abstract}

Copyright $\odot 2016$ Institute of Advanced Engineering and Science. All rights reserved.

\section{Corresponding Author:}

Min Raj Nepali,

Centre For Robotics Research,

Nitte Meenakshi Institute of Technology, Bangalore.

Email: nminraj92@gmail.com

\section{INTRODUCTION}

Immense work has been carried out on building robots which look alike human and are capable of mimicking few facial expressions [3]. MIT Lab has been actively involved in research regarding HRI [4]. Worldwide research is going on HRI and its best applicability in industrial and academic circle [6].

Humanoid Robotic Head is an exemplary of interdisciplinary work which strongly involves knowledge across various fields like mechanical design and analysis, control of motors, sensor interfacing and processing, computer vision, etc. The mechanical framework is designed by group of mechanical engineers and the control of motors is carried out by the Electrical and Electronics engineers whereas the development of GUI and the Computer vision part is carried out by Computer Science Engineers. The Project was carried out at Centre for Robotics Research, Nitte Meenakshi Institute of Technology (NMIT). Computer vision gives commands related to the different movements in real time, these commands are recognized by the control system which actuates the motors related to that movement which in turn move the links attached to those motors. And finally the different parts of the Robotic Head connected to that links are moved to mimic the different facial expressions. Well coordinated movements of the actuators are required in order to mimic the human facial expression in such a way that the desired message is conveyed to the observer. The closed loop control system incorporated for the Head ensures the well coordinated movement of the motors [5] [7]. For real time Expression, synchronization of the different actions is necessary which was taken care by thread programming. Memory and code block optimization was done to reduce the response time of the Robotic Head. The Embedded Board was selected in such a way that it was capable of handling all the aforementioned tasks subtly. The actuators involved here are dynamixel AX-18, MX-64 which were 
responsible for Head movement like up, down, right, left and opening of mouth. Modeling and proper mounting of these motors was required to achieve effective outcome [1] [2]. The other motors involved are Micro Servo Motors which are responsible for the movement of eyes in concerned directions such as left, right, up and down.

Dynamixel motors initially controlled by computer were ported to UDOO Board in which the linux environment was set up for the required interfacing and GUI was created using the Java (eclipse IDE) platform which was implemented on the same board [8][9] [10] [11]. So that the full fledged system is solely realized in the Embedded Board.

\section{SYSTEM OVERVIEW AND PLATFORM SPECIFICATIONS}

In this paper, we want to present the type of Control system used, its methodology, implementation steps with the UDOO Board and its various aspects, development of different modules, integration of various modules into a working module. In this section, development of the control algorithms, actuators, boards, communication protocols, and programming language will be discussed in elaborated view.

Closed loop Control system has been implemented in order to monitor the positions of the Dynamixel Motors. PID control has been incorporated to reduce the errors in Position Control. A database is created on the Processor as well as the controller part of the Board. This database defines the movements of the different parts like Neck, eyes, eyebrows, eyelids, etc which was invoked during the execution of the particular expression. To achieve the above mentioned task position control was incorporated. RS232 serial communication protocol has been implemented to share data and commands between different modules. These different modules involve GUI, Dynamixel control module, micro servo motor control module and voice playing module as shown in the Figure 1.

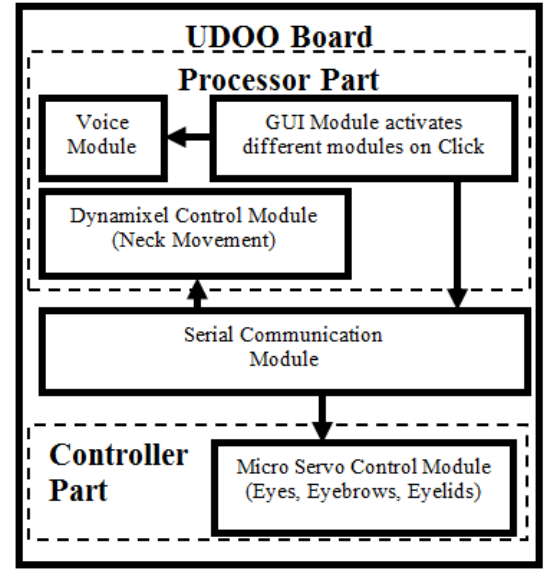

Figure 1. System Overview

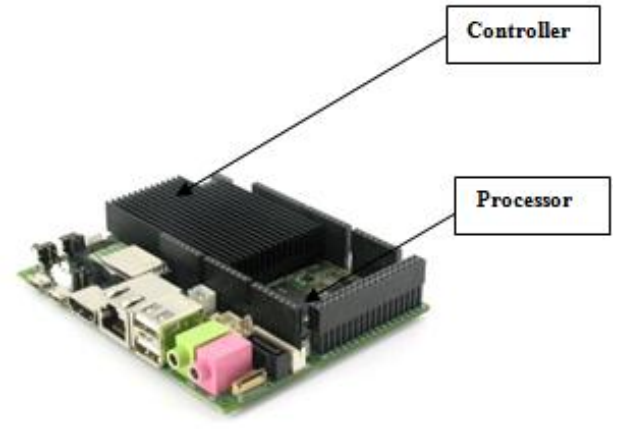

Figure 2. UDOO Quad Board

The complete system is realized using UDOO Board which has quad core A9 processor and a controller mounted on it as shown in Figure 2. It is capable of handling numerous tasks simultaneously. The processor mainly runs the GUI and Dynamixel commands and the controller takes care of the micro servo motors. Inter-processor communication is carried out to pass commands from Processor to the controller and monitor the output. The GUI is developed using java platform, the modules to control Dynamixel and Servo motors were developed using $\mathrm{C} / \mathrm{C}++$ and the modules which control micro-servo motors were written in Embedded C.

UDOO Board is a newly launched Board in the market on Kickstarter and marketed by Tenet Technotronics in India. It supports Linux as well as Android. We had installed Ubuntu which is a Linux based Operating system and which can be downloaded from UDOO Board home page [12]. Operating system supports all the programming languages and gives us numerous choices to implement our system.

\section{METHODOLOGY}

This section describes the various Modules, their functionality and developmental steps. 


\subsection{Development of GUI}

A flow chart summarizing the work flow of our proposed Graphical User Interface for Humanoid Robotic head is depicted in Figure 3 above.In order to start the process, user has to press initialization button to actuate motors, which requests user to enter the password. If password gets validated, then the user gets access permission to operate actions with Humanoid Robotic Head.

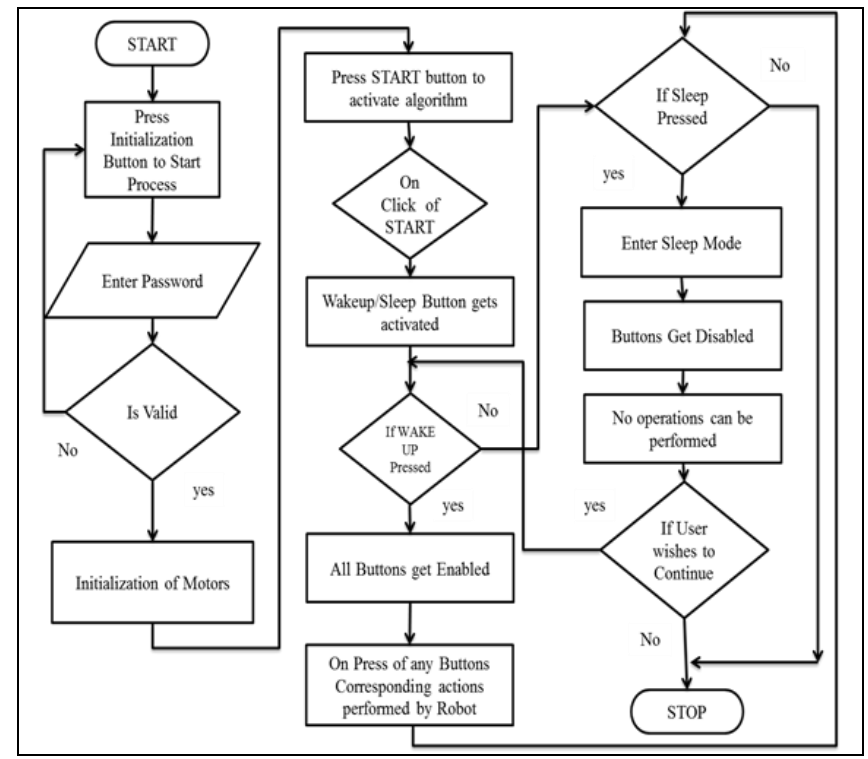

Figure 3. Process flow for the GUI

Second step is to press START button to begin Algorithm. Upon click of START button, WAKEUP/SLEEP button gets activated. By default, all the buttons except "specification" button will be disabled. On click of "wakeup" button, all other buttons gets enabled. And the Robotic head wakes up which indicates it is ready for use. On click of "I" button; the specifications of the robotic head will be displayed.

There are 22 action buttons. Among which, 6 buttons shows neck movements (Roll Left, Roll Right, Head Up, Head Down, Left, Right) 6 buttons (Laugh, Sad, Angry, Fear, Surprise, Tired) demonstrates expressions and other 10 buttons (Close Mouth, Open Mouth, Close Left Eye, Close Right Eye, Close Both Eye, Eye Ball Down, Eye Ball Up, Eyebrow Down, Eyebrow Up, Look side Ways) exhibit facial features of humanoid robotic head. Upon selection of each button, corresponding actions are invoked in the UDOO board and in turn initiates the motor movements of Robotic head.

\subsection{Control of Dynamixel motors and micro servo motors}

Assuming that Human Facial Expression and Neck movements are straight face expression, Position Control method has been implemented to actuate the motors to the required goal position and back to the original position. Home position is defined as the Normal position of human Head. The required goal position are predefined which are fed to the Dynamixel and micro servo control module.

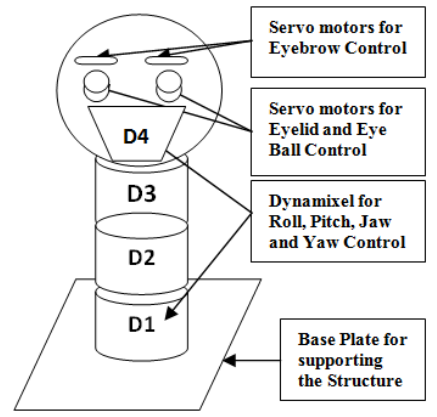

Figure 4. Placement of Motors to achieve different movement 
Dynamixel Motors are placed in a way as shown in Figure 4 whose combined movement give Roll, Pitch and Yaw movement to the Robotic Head. Movement of Dynamixel motors as well as micro servo motors was monitored using closed loop control system. Dynamixel motors are connected through USB2Dynamixel controller which is in fact controlled by the $\mathrm{C}$ program running on the Processor of the UDOO Board. Micro servo motors are directly controlled from the dedicated analog pins of the Controller part in UDOO Board. Libraries like dxl_sdk which are necessary to Control the Dynamixel motors are set up in the Processor of UDOO Board.

Servo Libraries are used to pass the servo commands form the Controller part.D1- Dynamixel 1 motor executes Yaw motion giving the Robotic Head turning action.D2-Dynamixel 2 motor executes Roll motion facilitating the Robotic Head for rolling action.D3-Dynamixel 3 motor executes Pitch motion providing the Robotic Head up and Down motion.D4-Dynamixel 4 motor helps in Jaw movement by moving the jaw up and down that make Robotic Head capable of talking. Similarly, eight Micro servo motors are attached to the Eyeball, Eyelid and Eyebrow. These micro servo motors execute actions related to Visual expressions.

\subsection{Communication between different Modules}

Different modules communicate with each other in order to carry out the job smoothly. GUI module passes commands to the Dynamixel module as well as the micro servo motors. To Dynamixel motors, the GUI passes commands directly through the $\mathrm{C}$ program that runs in the processor part whereas to micro servo motors GUI first passes commands to processor and processor then passes commands to the Controller via inter processor communication module which is inbuilt in the UDOO Board as demonstrated in the Figure 5.

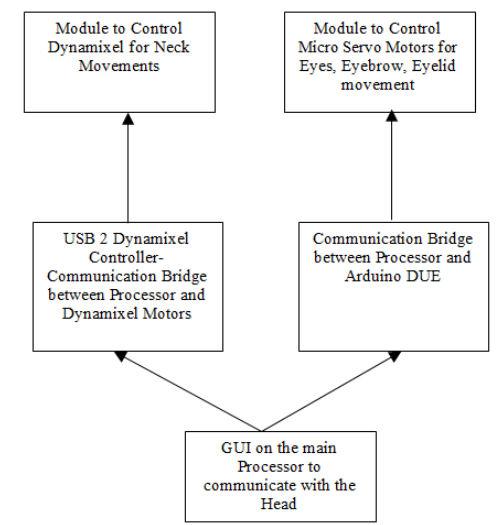

Figure 5. Different Communication Modules and Communication Process between them

\section{RESULTS}

Figure 6 (a) shows the various buttons named after various Human Facial Expressions. Figure 6 (b)(c) show the activation of buttons after pressing the start button, which now user can press to demonstrate any Expression. Figure 6 (d) shows the specification of the Robotic Head such as degrees of freedom, capabilities like Roll Pitch Yaw, Design and Analysis of the Robot, etc.

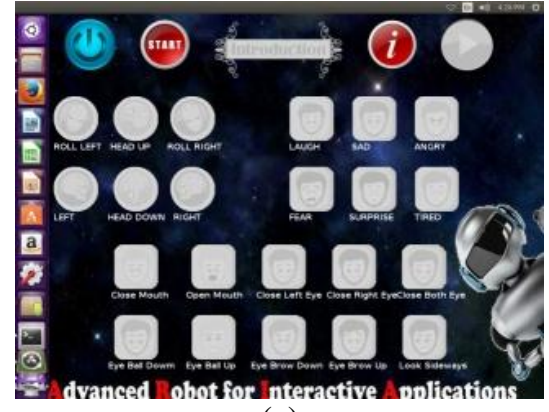

(a)

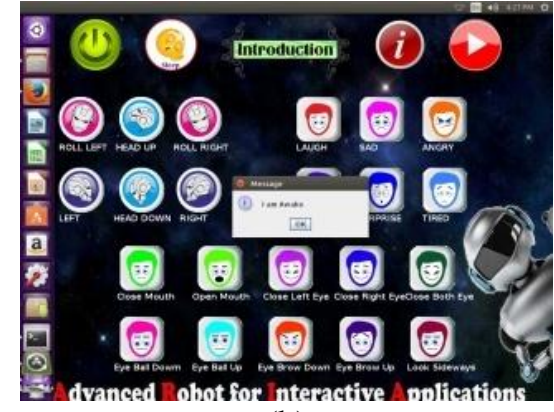

(b) 


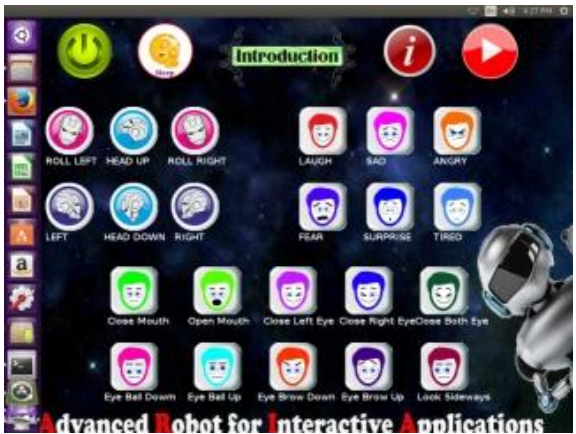

(c)

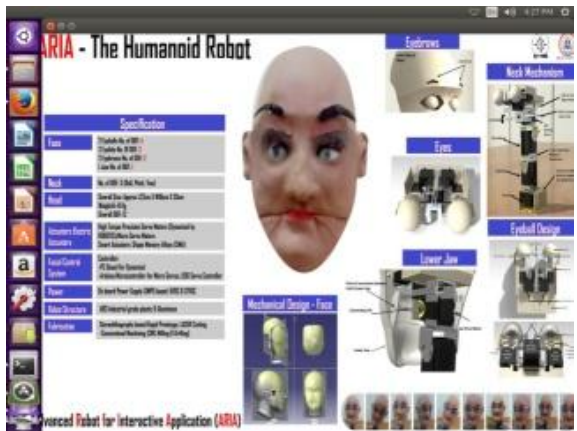

(d)

Figure 6 : (a) (b) (c) (d) Different windows of GUI

\begin{tabular}{|l|l|l|}
\hline Real Time Expression & \multicolumn{1}{|c|}{ Actuators } & \multicolumn{1}{c|}{ Degree of Rotation } \\
\hline \multirow{2}{*}{} & ServoM1 & 0 \\
\cline { 2 - 3 } & ServoM2 & 0 \\
\cline { 2 - 3 } & ServoM3 & 0 \\
\cline { 2 - 3 } & ServoM4 & 0 \\
\cline { 2 - 3 } & ServoM5 & 0 \\
& ServoM6 & 0 \\
& ServoM7 & +40 \\
& ServoM8 & -30 \\
& MX64_1 & Home Position \\
& MX64_2 & Home Position \\
& AX18_1 & Home Position \\
\hline
\end{tabular}

Figure 7 : Robotic Head Demonstrating the Roll Left Movement

\begin{tabular}{|l|l|l|}
\hline Real Time Expression & \multicolumn{1}{|c|}{ Actuators } & \multicolumn{1}{c|}{ Degrees of Rotation } \\
\hline \multirow{2}{*}{} & ServoM1 & 0 \\
\cline { 2 - 3 } & ServoM2 & 0 \\
\cline { 2 - 3 } & ServoM3 & 0 \\
\cline { 2 - 3 } & ServoM4 & 0 \\
\cline { 2 - 3 } & ServoM5 & 0 \\
& ServoM6 & 0 \\
& ServoM7 & +40 \\
& ServoM8 & -30 \\
& MX64_1 & Home Position \\
& MX64_2 & Home Position \\
& AX18_1 & Home Position \\
& AX18_2 & Home Position \\
\hline
\end{tabular}

Figure 8 : Robotic Head Demonstrating the Normal Facial Expression

\begin{tabular}{|l|l|l|}
\hline Real Time Expression & Actuators & \multicolumn{1}{c|}{ Degree of Rotation } \\
\hline \multirow{4}{*}{} & ServoM1 & 0 \\
\cline { 2 - 3 } & ServoM2 & 0 \\
\cline { 2 - 3 } & ServoM3 & 0 \\
\cline { 2 - 3 } & ServoM4 & 0 \\
\cline { 2 - 3 } & ServoM5 & +20 \\
& ServoM6 & 0 \\
& ServoM7 & -30 \\
& ServoM8 & +30 \\
& MX64_1 & Home Position \\
& MX64_2 & Home Position \\
& AX18_1 & Home Position \\
& AX18_2 & Home Position \\
\hline
\end{tabular}

Figure 9. Robotic Head showing the Angry Face 
Figure 7 shows the Robotic Head in Normal condition Figure 8 shows the Robotic Head making Angry Face Figure 9 shows the Robotic Head executing Roll Left command.

\section{CONCLUSION AND FUTURE SCOPE}

The Robotic Head discussed in this paper is a prototype which is basically dedicated to exhibit various possible Human Facial Expressions. The Head basically sows fixed and predefined movements. It does not involve the dynamics involved in Human Facial Expressions. Also it does not show any sort of intelligence. It basically can be considered as a platform to study various possibilities and applicability of Human Robot Interaction in various others fields.

The Robotic Head can be made intelligent by adding Robust Vision System. Materials like bio sensors and shape memory alloys (SMAs) can be used to fabricate the Robotic Head which exhibits more degrees of freedom allows to realize Dynamics of Human Robot Interaction in a precise way. The Robotic Head can be incorporated with various other Algorithms and can be upgraded to carry out following Applications.

[1] Web-Mining, where the Robotic Head will login into the internet, search for the user defined product, its review (pros and cons) and gives us the feedback regarding the product.

[2] Leaf identification, where the Robotic Head will identify whether the leaf is healthy or not, if the leaf is unhealthy the Robot will tell how much the leaf is affected by the disease.

[3] Activity Tracking, where robot will find out movement in the environment and inform whether the object of consideration is moving slow or fast.

[4] Sentence recognization and reading out the sentence from a wall or a board.

\section{ACKNOWLEDGEMENT}

Our sincere thanks goes to the Vision Group of Science and Technology (VGST), Karnataka to acknowledge our research and provide the financial support to carry out the research work at NMIT. We express our thanks to our colleagues at the Robotics Research Center, NMIT who made significant contribution in this multidisciplinary research work.

\section{REFERENCES}

[1] Prof. Dr. Ir. S. Stramigioli, ir. E.C. Dertien, ir. G. van Oort, "Characterization and modeling of a Dynamixel servo", University of Twente, Report nr. 035CE2008, November 2008

[2] Data sheet for dynamixel motor in section of technical support http://support.robotis.com/en/techsupport_eng.htm\#product/dynamixel/mx_series/mx-28.htm

[3] Noriaki Mitnunaga, Christian Smith, Takayuki kanda, Hiroshi Ishiguru, NorihiroHagita, "Robot Behavior Adaptation for Human-Robot Interaction based on policy gradient Reinforcement Learning", Intelligent Robots and Systems, 2005. (IROS 2005). 2005 IEEE/RSJ, Date of Conf. 2-6 Aug. 2005, pages 218 - 225

[4] Cory D. Kidd,"Human-Robot Interaction: Recent Experiments and Future Work", MIT Media Lab.

[5] KarstenBerns, JochenHirth, "Control of facial expressions of the humanoid robot Head ROMAN", Intelligent Robots and Systems, 2006 IEEE/RSJ, Date of Conf. 9-15 Oct. 2006, pages 3119-3124.

[6] MarenBennewitz, Felix Faber, Dominik Joho, Michael Schreiber, and Sven Behnke, "Enabling a Humanoid Robot to Interact with Multiple Persons", DARH2005 Conference, Session 3.4

[7] YonasTadesse, ShashankPriya," Analysis and Design Method: An Approach to Determine Humanoid Skin Deformation", Transactions of the ASME, 021010-4 / Vol. 4, MAY 2012

[8] UDOO

Manual: http://udoo.org/download/files/Documents/UDOO_Starting_Manual_beta0.4_11_28_2013.pdf

Starting

[9] Schematics: http://udoo.org/download/files/schematics/UDOO_REV_D_schematics.pdf

[10] UDOO Pinout: http://udoo.org/download/files/pinout/Udoo_pinout_diagram.pdf

[11] Datasheet: http://www.seco.com/misk/UDOO_datasheet.pdf

[12] http://elinux.org/UDOO 